\title{
3D RECONSTRUCTION OF THE ANCIENT EGYPTIAN MUMMY SKELETON FROM THE PUSHKIN STATE MUSEUM OF FINE ARTS (I,1 1240)
}

\author{
S.V. Vasilyev ${ }^{3}$, O.A. Vasilyeva ${ }^{4}$, R.M. Galeev ${ }^{3}$, O.P. Dyuzheva ${ }^{4}$, M. Novikov ${ }^{2 *}$, I.A. Chichaev ${ }^{1}$, E.B. Yatsishina ${ }^{1}$ \\ ${ }^{1}$ National Research Center Kurchatov Institute - chichaev.ilya@gmail.com, yacekaterina@yandex.ru \\ ${ }^{2}$ Research Center Crystallography and Photonics RAS, Shatura, Russia - novikov@ rambler.ru \\ ${ }^{3}$ Institute of Ethnology and Anthropology RAS, Moscow, Russia - vasbor1@yandex.ru, ravil.galeev@gmail.com \\ ${ }^{4}$ The Pushkin State Museum of Fine Arts - vassilievagmii@gmail.com, olga.dyuzheva@arts-museum.ru
}

\section{Commission II, WG II/10}

KEY WORDS: Paleoanthropology, Mummies, Non-contact measurements, Digital model, Additive technologies, Computed tomography

\begin{abstract}
:
The aim of this study was to obtain a non-destructive three-dimensional model of the ancient Egyptian mummy skeleton from the collection of the Pushkin State Museum of Fine Arts (I,1 1240). The objective of this research was to study the possibility of precise segmentation of bone structures and their separation from the surrounding mummified soft tissues, as well as the reconstruction of destroyed bone fragments according to the preserved shape. With the help of additive technology made all fragments of the skeleton, including the distal phalanx. The digital models and plastic reconstructions of historical artifacts can be used for further research, for example, the biomechanical and morphometric ones, as well as for the purpose of information and education, or as a supplement to the original museum objects.
\end{abstract}

\section{INTRODUCTION}

Nowadays, the traditional approaches to obtaining historical information from the narrative and archaeological sources are significantly supplemented by the advanced natural science research methods. The concept of digital or virtual paleontology has been formed on the basis of the application of the new information technologies (Cunningham J. et al, 2014). The first CT scan of a mummy was performed in 1977 by Derek Harwood-Nash in Toronto, Canada only about four years after the clinical introduction of the CT scanner (Harwood 1979). Harwood-Nash performed a CT scan on the desiccated brain of a boy and the mummy of a young woman, having demonstrated that X-ray CT scanning was a useful tool in archaeology. In recent times, the methods of computer and magnetic resonance tomography have been used to study the mummification process in a non-invasive way, to establish sex and age, to obtain anthropological characteristics, as well as to conduct forensic and medical examination of ancient mummified objects (Zesch S. 2016). Three- dimensional models and reconstructions of historical artifacts are frequently applied in the modern science. They allow visualizing various pathologies and conducting the anthropometric studies of the mummified remains without destroying the original object (Davies et al 2017).

This paper describes a part of the interdisciplinary study of ancient Egyptian mummies. Combining the experience of studying the collection of ancient Egyptian art from the Pushkin State Museum of Fine Arts with the technological experimental infrastructure of the National Research Center "Kurchatov Institute" (Koval"chuk M. 2016), as well as the research institutes of the Russian Academy of Sciences, including in the field of additive technologies and anthropology, allowed us to improve the already

\footnotetext{
${ }^{*}$ Corresponding author
}

developed methods and to offer the new ways of extracting hidden information from the archeological sources.

\section{3D RECONSTRUCTION OF THE ANCIENT EGYPTIAN MUMMY SKELETON}

\subsection{The object of the study}

A very informative source on the history and culture of ancient Egypt are the mummies, a comprehensive study of which is now becoming increasingly important (Cesarani F. 2003). The art of mummification was one of the important indicators of the level of technology, culture and religion of Ancient Egypt. In the Russian Federation, a collection of nine mummies (Fig. 1) is being kept at the Pushkin State Museum of Fine Arts (Moscow)

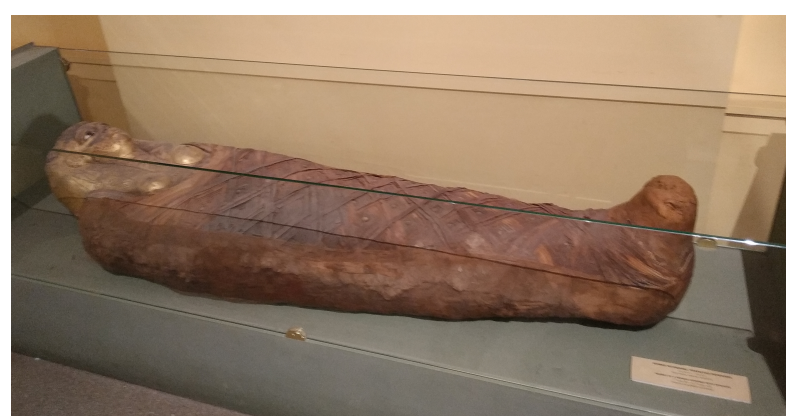

Figure 1. View of the permanent display of Room 6 in the Pushkin Museum

A part of the collection was acquired in 1909 from Russian egyptologist Vladimir Golenischev and was later expanded. Now it includes 9 mummies dated from I Mill. BCE till I-III cent. CE. 
The object of this study was the ancient Egyptian mummy (I,1 1240) from the collection of the Pushkin State Museum of Fine Arts (Table 1).

\begin{tabular}{lll}
\hline \multicolumn{2}{l}{ NumbeName } & Description \\
\hline I,1a & Mummy of a & Upon the face is a gilded \\
1240 & $20-25$ years & mask with inlaid glass \\
& old woman, in & eyes. Characteristic of the \\
wrapping, with & $\begin{array}{l}\text { Graeco- Roman period } \\
\text { a cartonnage }\end{array}$ & is an elaborate wrapping \\
mask II III & arranged in geometrical \\
centuries CE. & $\begin{array}{l}\text { pattern, decorated with } \\
\text { gilded plaques. }\end{array}$ \\
\hline
\end{tabular}

Table 1. Description of the mummy

\subsection{The goals of the study}

Three-dimensional visualization of the mummy skeleton is important for the paleontological research and museum exhibition projects. A three-dimensional model can be used for detailed anthropological measurements of mummy skeletons and skulls, clarification of their sex, age, congenital or acquired abnormalities, as well as for medical studies of existing injuries, determining possible causes of death. A plastic copy of the mummy skull allows us to visualize the appearance of inhabitants of the Nile valley without destroying the mummy.

\subsection{The methods of the study}

The modern technologies, such as computed tomography, magnetic resonance imaging and others, allow us to study artifacts in a non-destructive way on a fundamentally new scientific level (Moissidou D. et al. 2015). In this work, the technological infrastructure of the NRC "Kurchatov Institute" provided the tomography scans of some intact Egyptian mummies and their fragments, as well as the animal mummies from the collections of the Pushkin Museum (Yacishina E.B. 2018). Tomographic scanning was performed using the X-ray PET-CT scanner Biograph mCT$\mathrm{S}(40)$ by Siemens (Figure 2).

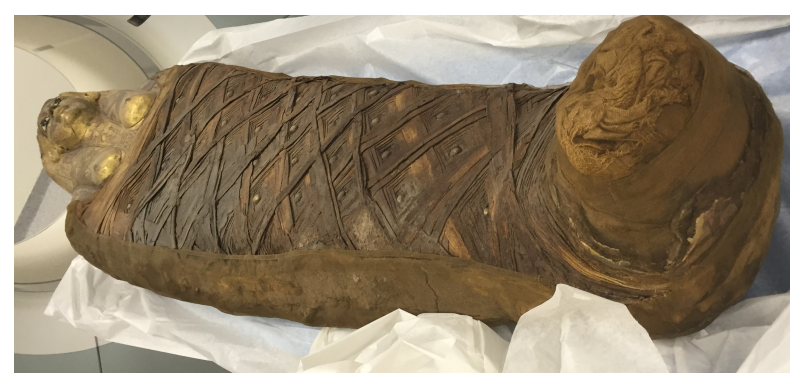

Figure 2. CT scan of the Egyptian mummy (I,1 1240)

As it has already been noted in some studies (Cox S. 2015), the choice of CT scan parameters determines the quality of the data received. For transportation and protection of the mummified specimens while scanning the special containers were constructed. In the process of research, the optimal scanning mode (voltage and current on the X-ray tube for CT) was determined in order to obtain the most informative parameters of the tomographic images for $3 \mathrm{D}$ reconstruction. According to the results of the research, the optimal scanning parameters of $140 \mathrm{kV}$ and 120 A were selected for further work. Additionally, two modes of image reconstruction were used, differing in sensitivity of contrasting to bone and soft tissues with a voxel size of $0.3 \mathrm{~mm} * 0.5 \mathrm{~mm}$
* $0.5 \mathrm{~mm}$. The study allowed us to get the most accurate data to obtain a three-dimensional reconstruction in a non-destructive way.

The obtained tomographic scanning data allow us to create the three-dimensional models of the skeleton for detailed anthropological measurements, to determine sex and age, to perform the reconstruction, to use the results for the visualization.

\subsection{Complex anthropological description of the mummy}

The mummy I, 11240 is that of a 20-25 years old woman. She lies on her back, the skull is slightly turned to the left, the bones of the hands rest parallel to the pubic joint, touching each other; the bones of the left hand are extended, the main phalanxes of the right hand are extended too and the middle ones are bent; the bones of the legs are extended, the knee-joints come in touch with each other by their inner sides; the feet bones as well, the feet are slightly extended.

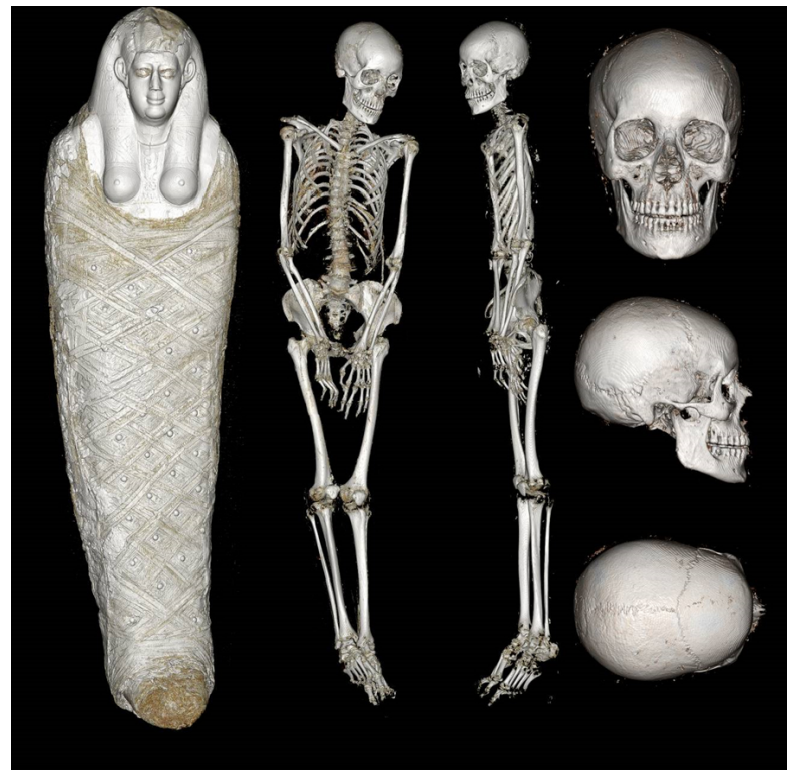

Figure 3. Mummy I,1a 1240. From left to right: the top level of the visualization; the skeleton position (frontal norm); the skeleton position (lateral norm); three basic norms of the skull

The craniological characteristics certainly show that the mummified human belonged to a variant of the Mediterranean anthropological type of Caucasians. This type is characterized by mesocrania, gracility, narrowness, horizontal profiling and a relatively high and narrow nose. A study of the proportions of the limbs revealed a relatively elongated tibia, which can be considered as a manifestation of adaptation to the hot climate. The woman was characterized by rather narrow shoulders and a very wide and low pelvis. The lifetime body length was $153.3 \mathrm{~cm}$, which correlates with the average height at that time. The humerus and the clavicle are medium massive, the remaining bones, and both the arms and the legs are gracile. The tibia is relatively dilated in the diaphysis. The muscular arm relief is poorly developed, the muscular leg relief is moderately pronounced. There are signs of a multilevel spinal injury, as well as a rump fracture (Figure 4).

The upper cortical of the body of the 2nd lumbar vertebra on the right is fractured (indicated by the white arrow). The trauma can probably date back to the persons lifetime as a result of a fall from the height. 


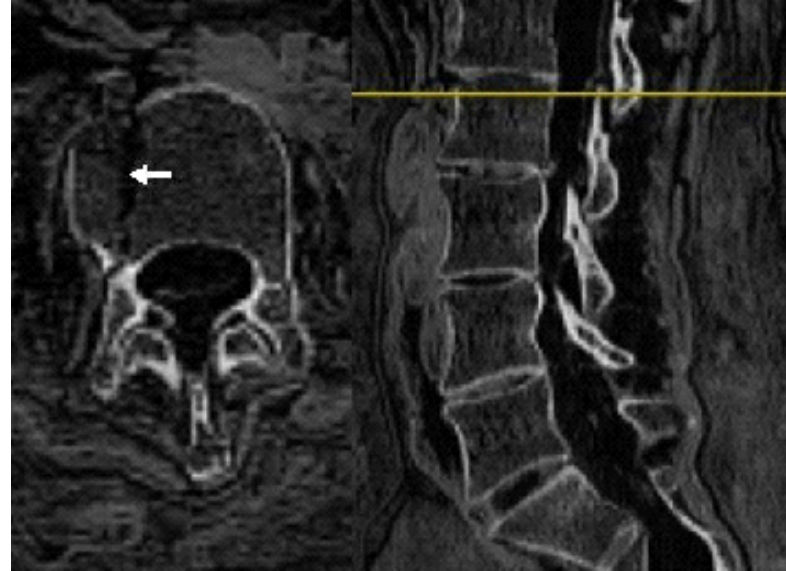

Figure 4. CT scan of the lumbar spine of the mummy I,1a 1240.

\subsection{Virtual reconstruction}

At the next stage the digital models of the skeleton and body parts for a virtual three-dimensional reconstruction were created (Hegna T. et al 2016). The results of CT data in DICOM format [DICOM] were transmitted into ILIT RAS via the electronic network. To process the tomograms and construct the 3D models of the bone skeleton, the computer program Mimics from Materialise was used. The experience of manufacturing medical models (Chereby'lo S. 2015) according to the tomographic data showed that the object of study (for example, a human skull) is a rather complex formation with a large number of internal cavities, which consists of the biological tissues of different density. At the same time, a three-dimensional solid model reproduces an object of only one density, so it is very important to choose a cut-off density threshold (i.e. a certain brightness value on the tomogram), which determines the result, when all the areas of the given or higher density become solid, and the areas of the reduced density are cut off.

To quantify the density of the CT structures a special X-ray attenuation scale, called the Hounsfield scale, is being used (it is visually reflected on the monitor in black and white image spectrum). The range of scale units (i.e. densitometric indicators) corresponding to the degree of attenuation of X-ray radiation by the anatomical structures of the body keeps in the interval from -1024 to +3071 . The zero value in the Hounsfield scale corresponds to the density of water, the negative values of the scale correspond to air and adipose tissue, the positive ones - to soft tissues and bone tissue. The task of segmentation of the given area and the selection of the required structure involves the removal of all unnecessary characteristics (Friedman S. 2012).

First of all, the threshold values of the density of the secreted tissues are established and in this case two analytical mechanisms are used. The first one is the voxel visualization. In the original tomographic image, all voxels (i.e. image units) store the density value, but for a segmented display, some color and transparency value can be assigned to each voxel. There for the transfer function $\mathrm{T}(\mathrm{I})$ is responsible, which sets the value of the color range in accordance with the specific density I. Since the stored density values are actually integers, the function $\mathrm{T}$ (I) can be given in the tabular form and in this case it is called the color table. Due to the selected color table, the three-dimensional reconstruction will look different: the densities of one range, corresponding to one type of fabric, can be hidden, and the densities of other ranges, on the contrary, can be made visible and painted in the selected color. Thus, different color tables allow us to visualize different organs and tissues, or the same organs in a different way.

This mechanism permits the certain structures to be segmented. The color table and the current density window serve as the parameters determining one of the segmentation algorithms. Another parameter defining the current segmentation is the visibility mask. In this mask, each voxel corresponds to one bit, which, respectively, can have the following values: 0 - the voxel is deleted, 1 - the voxel is not deleted. Thus, the tools that remove excess tissue simply set the mask to zero for the voxels to which they are applied. When using a visibility mask, it becomes possible to hide organs and tissues regardless of the color table selected for the visualization. Thus, the current segmentation is determined by three parameters: a color table, a density window, and a visibility mask. If any of these parameters changes, the segmentation may also change. The segmentation result is also presented in the form of a bit mask - a single value of a voxel mask means that it belongs to the segmented object, and a zero value, respectively, has a reverse meaning - the voxel does not belong to the segmented object.

\subsection{Basic segmentation tools}

Polygon cutting. The tool is applicable to three-dimensional reconstruction. Two options are possible: delete everything that has fallen into the selected area, or, on the contrary, delete everything around the selected area.

Remove visible tissue with an eraser in the form of a ball or cylinder. The advantage of this tool is its locality. In addition, this tool can be used on two-dimensional reconstruction slices and perform manual segmentation of the desired structure. Selection of related areas. For example, you can remove a single bone that is not visually in contact with others, or, alternatively, select it from other structures. For this purpose, the concept of the minimum thickness of a structure that makes an object connected, i.e. if the two parts of the object are connected by some structure, the thickness of which is less than the specified value, then these two parts will not be considered connected.
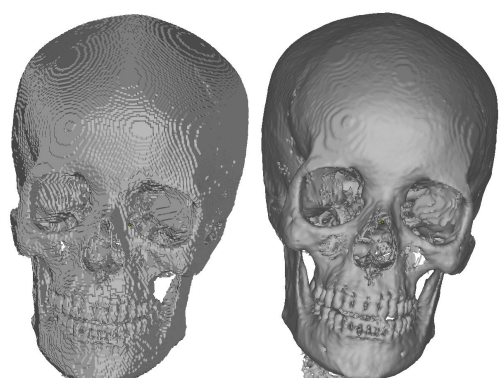

Figure 5. The result of smoothing the voxel model

A segmented structure is a saved result of segmentation that can be edited and visualized independently. Based on any segmented structure, it is possible to construct its digital model approximation of the surface of a segmented structure by polygonal elements (triangles). This is one of the most important segmentation results, since the export of segmentation results in the form of a polygonal model allows you to work with these results in most 3D modeling programs. To build grids, the marching cubes algorithm is used, which is both simple and the most effective for this task. Since the voxel model consists of cubic voxels, when approximating its surface, this cubicity is preserved and the grid 
looks graded. Smoothing is performed by the Laplace method, which consists in the fact that the coordinates of each vertex of the grid are replaced by the averaged coordinate of all the neighboring vertices of Figure 5.

The main task of this stage was the selection of the correct and most informative level of tomographic image conversion into a three-dimensional model (Figure 6). At the standard densities of 266 (HU scale), the image and the model contain noises and artifacts, which are significantly reduced at 400 .
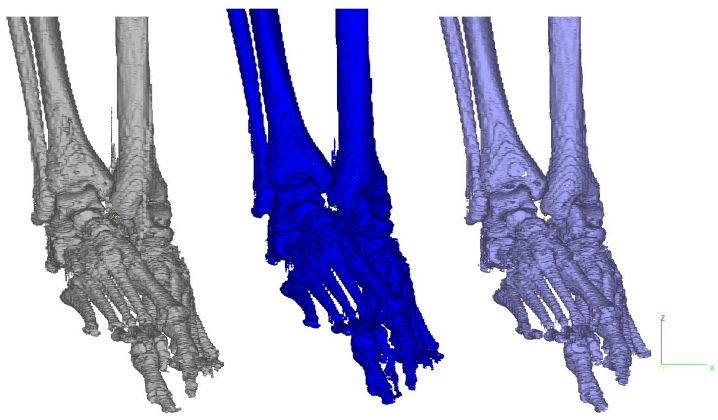

Figure 6. Selection of the bone density level (HU) to convert the image to a three-dimensional model. A) 226 B) 300 C) 400

But in this case a part of skeleton bones can be lost, and it is necessary to draw the tomography images for restoration of the lost fragments by hand (Figure 7).

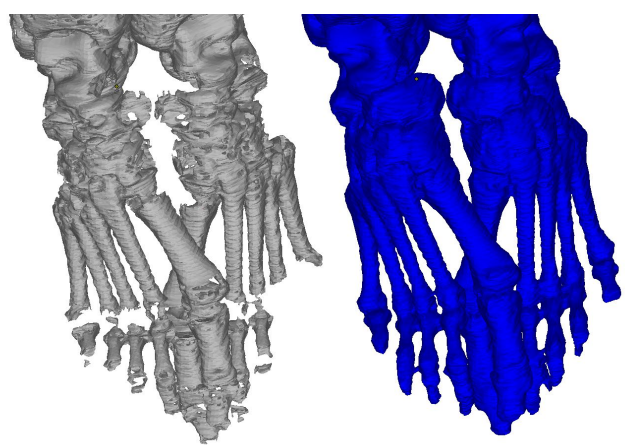

Figure 7. Reconstruction of the fragments by manual drawing of the image. A) initial model. B) three-dimensional model with reconstructed fragments.

While scanning, a large amount of tomographic data (5661 slices) was obtained, which was divided into 4 zones: the head section, the torso, the pelvic section from the upper part of the legs to the lower part of the legs with feet. A sequential processing of the tomograms of each individual zone was carried out and its three-dimensional model was built. At the next stage, the threedimensional models of individual zones were combined (Figure 8).

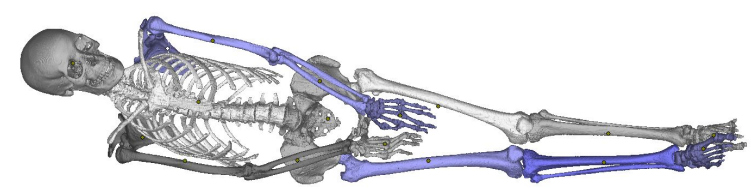

Figure 8 . The united three-dimensional model of all the skeleton fragments

\subsection{Additive manufacturing}

At the last stage, the production of separate fragments of the skeleton and body parts for the realistic three-dimensional reconstruction was carried out by means of additive technologies.

Each 3D printing technology has its own strengths, weaknesses, and requirements, and is suitable for different applications and businesses. The following table 2 summarizes some key characteristics and considerations.

\begin{tabular}{llll}
\hline & FDM & SLA & SLS \\
\hline $\begin{array}{l}\text { Reso- } \\
\text { lution }\end{array}$ & $\oplus \oplus \oplus \ominus \ominus$ & $\oplus \oplus \oplus \oplus \oplus$ & $\oplus \oplus \oplus \oplus \oplus$ \\
\hline $\begin{array}{l}\text { Accu- } \\
\text { racy }\end{array}$ & $\oplus \oplus \oplus \oplus \ominus$ & $\oplus \oplus \oplus \oplus \oplus$ & $\oplus \oplus \oplus \oplus \oplus$ \\
\hline $\begin{array}{l}\text { Surface } \\
\text { finish }\end{array}$ & $\oplus \oplus \oplus \ominus \ominus$ & $\oplus \oplus \oplus \oplus \oplus$ & $\oplus \oplus \oplus \oplus \ominus$ \\
\hline $\begin{array}{l}\text { Through- } \\
\text { put }\end{array}$ & $\oplus \oplus \oplus \oplus \ominus$ & $\oplus \oplus \oplus \oplus \ominus$ & $\oplus \oplus \oplus \oplus \oplus$ \\
\hline $\begin{array}{l}\text { Complex } \\
\text { Designs }\end{array}$ & $\oplus \oplus \oplus \ominus \ominus$ & $\oplus \oplus \oplus \oplus \oplus$ & $\oplus \oplus \oplus \oplus \oplus$ \\
\hline $\begin{array}{l}\text { Ease of } \\
\text { Use }\end{array}$ & $\oplus \oplus \oplus \oplus \oplus$ & $\oplus \oplus \oplus \oplus \oplus$ & $\oplus \oplus \oplus \oplus \ominus$ \\
\hline Pros & $\begin{array}{l}\text { Fast, Low- } \\
\text { cost machines } \\
\text { and materials }\end{array}$ & $\begin{array}{l}\text { Great value, } \\
\text { High } \\
\text { racy, Smooth- } \\
\text { surface finish }\end{array}$ & $\begin{array}{l}\text { Design for free- } \\
\text { dom no need } \\
\text { support } \\
\text { structures }\end{array}$ \\
\hline Cons & $\begin{array}{l}\text { Low accu- } \\
\text { racy, Limited } \\
\text { design com- } \\
\text { patibility }\end{array}$ & $\begin{array}{l}\text { Average build } \\
\text { volume }\end{array}$ & $\begin{array}{l}\text { Rough sur- } \\
\text { face finish }\end{array}$ \\
& &
\end{tabular}

Table 2. Comparison of FDM, SLA, and SLS Technologies

Three-dimensional printing technology was chosen technology CJP (model zPrinter 650) printing of fine gypsum powder, followed by sizing the model with glue based on cyanoacrylate (fig.9 a). The volume of the 3D model of the skull was 0.490 liters. The cost of the materials used is approximately 25000 rubles. The time of three-dimensional printing was 11 hours, the subsequent cleaning and surface treatment took 3 hours, the time of impregnation of the printed skull with cyanoacrylate and its subsequent drying was another 2 hours, total for the production of one skull was spent 16 hours. As the other additive technologies have been used laser stereolithography, apparatus LC400 developed in ILIT RAS (Figure 9b).

With the help of additive technologies, the skull of the mummy was made for the subsequent reconstruction of the appearance by the method of Gerasimov (fig.9 c). A SLA copy was chosen for this, as it has a higher surface quality and is better processed. The three-dimensional models of all mummy were made after special processing on a laser stereolithograph (Nedden D. 1994) from photopolymers in ILIT RAS (Figure 10).

The total volume of the entire model was 4.5 liters; the total production time took about 140 hours. In the future, a full-scale copy of the entire skeleton of the ancient Egyptian mummy can be moved to the Pushkin State Museum of Fine Arts (Moscow) for the morphological and medical research, and probably put on display. 


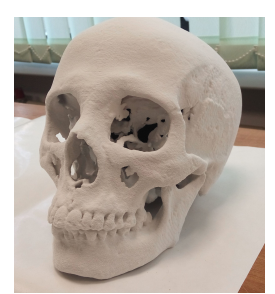

(a) zPrinter

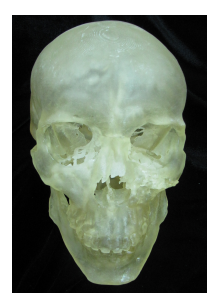

(b) laser stere- (c) reconstruction of olithography the appearance
Figure 9. A plastic copy of the three-dimensional skill of mummy reconstruction (a) zPrinter, (b) laser stereolithography, (c) reconstruction of the appearance

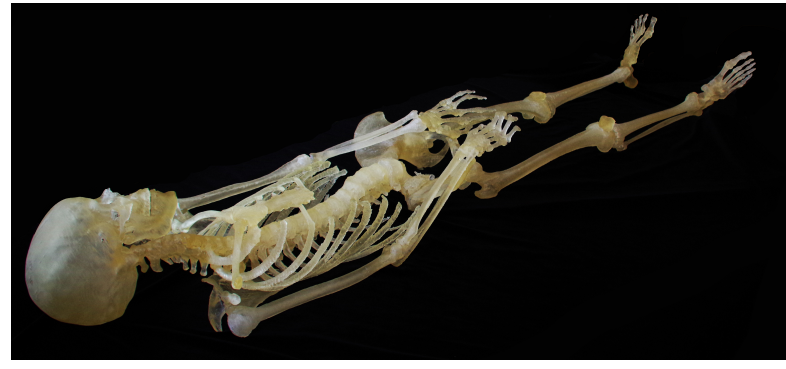

Figure 10. A plastic copy of the three-dimensional mummy reconstruction made by the laser stereolithography

\section{CONCLUSION}

The development of methods for creating the digital models of real objects and the use of additive technologies made it possible to create the high-precision and realistic prototypes of the valuable historical artifacts. The digital models and plastic reconstructions can be used for further biomechanical and morphometric research, as well as for informative and educational purposes or as a supplement to the museum display. The results of such work may contribute to the historical, anthropological, cultural, and museological studies.

\section{ACKNOWLEDGEMENTS}

The study was performed with financial support of the RFBR, Research project No. 17-29-04144 OFI-m.

\section{REFERENCES}

Cesarani F., 2003. Whole-body Three Dimensional multidetector CT of 13 Egyptian Human Mummies // American journal of Roentgenology. V. 180. N. 3. P. 597606., 2003

Chereby'lo S.A., Evseev A.V., Ippolitov E.V., Novikov M.M., 2015, Primenenie lazernoj stereolitografii v medicine v monografii Sovremenny'e lazerno- informacionny'e texnologii, pod red. V.Ya. Panchenko i F.V.Lebedeva, M.: Interkontakt Nauka, 358-373, ISBN 978-5- 902063-52-0.

Cox Samantha L., 2015. A critical look at mummy CT scanning. The anatomical record. Vol. 298. Pp. 10991110.

Cunningham John A. et al, A virtual world of paleontology, Trends in Ecology \& Evolution, Vol. 29, No. 6, pp. 347-357. 2014
Davies TG et al. 2017, Open data and digital morphology, Proc. R. Soc. B 284:20170194 http://dx.doi.org/10.1098/rspb.2017.0194

Digital Imaging and Communications in Medicine (DICOM), National Electrical Manufacturers Association, Rosslyn, USA,https://www.dicomstandard.org/current/.

Friedman, Saul et al. 2012, Computed Tomography (CT) Bone Segmentation of an Ancient Egyptian Mummy A Comparison of Automated and Semiautomated Threshold and Dual-Energy Techniques. Journal of computer assisted tomography. 36. 616-622. 10.1097/RCT.0b013e31826739f5. http://dx.doi.org/10.1155/2015/192829

Koval'chuk M.V., Yacishina E.B., Blagov A.E., Tereshhenko E.Yu., Prosekov P.A., D‘yakova Yu.A. 2016, Rentgenovskie i sinxrotronny"e metody" $\mathrm{v}$ issledovaniyax ob"ektov kul 'turnogo naslediya. // Kristallografiya, T. 61, No 5, s. 681-690

Moissidou D. et al. 2015, Invasive versus Non Invasive Methods Applied to Mummy Research: Will This Controversy Ever Be Solved, Hindawi Publishing Corporation, BioMed Research International Article ID 192829, 7 p

Nedden D.N. et al. 1994, Skull of a 5300-year-old Mummy: Reproduction and Investigation with CT-giuded Stereolithography// Radiology, V. 193. P. 269.

Posh J.C. Digital Data Recording and Interpretational Standards in Mummy Science // The Anatomical Record. 2015, V. 298. P. 1116.

Zesch S. et al. 2016, From First to Latest Imaging Technology: Revisiting the First Mummy Investigated with X-ray in 1896 by Using Dual-Source Computed Tomography // European Journal of Radiology V.3. P. 172

Yacishina E.B., Koval'chuk M.V., Loshak M. D., Vasil'ev S.V., Vasil'eva O.A., Dyuzheva O. P., Pozhidaev V.M., Ushakov V.L. 2018, Mezhdisciplinarny'e issledovaniya egipetskix mumij iz kollekcii gosudarstvennogo muzeya izobrazitel'ny 'x iskusstv im. A.S. Pushkina v nacional'nom issledovatel 'skom centre Kurchatovskij institut. Kristallografiya, tom 63, No 3. S. 479-490

D.F.C. Harwood-Nash, Computed tomography of ancient Egyptian mummies, Journal of Computer Assisted Tomography 3:768773. 1979.

Hegna Thomas A., Johnson Robert E. 2016, Preparation of fossil and osteological 3d-printable models from freely available ctscan movies, Journal of Paleontological Techniques, Number 16, 1-10. 2016 